\title{
Analysis of Electric Field and Current Density on XLPE Insulator
}

\author{
M. H. M. Sharif, N. A. M. Jamail, N. A. Othman, M. S. Kamarudin
}

Faculty of Electrical and Electronic Engineering, Universiti Tun Hussein Onn Malaysia (UTHM), 86400 Batu Pahat, Johor, Malaysia

\begin{abstract}
Article Info
Article history:

Received Aug 22, 2017

Revised Nov 52017

Accepted Nov 15, 2017

Keyword:

Current density

Electric field

Quickfield

Void

XLPE

ABSTRACT

Good condition and size of the insulator are important to ensure the excellent electric field and current density performance because of aging and degradation HVDC cable problem. At present, the existing insulator which is XLPE insulator that had been used does not meet the capabilities criteria of HVDC cable due to the problem in terms of the condition of the insulator which is the presence of a void in the insulator. A research had been made with Quickfield Software to calculate the electric field and current density in the XLPE insulator of HVDC cable. A void is created in the XLPE insulator by using Quickfield Software by varying diameter of void and distance of void from conductor. XLPE insulator is taken as main research object. The results show that largest diameter of void and nearest distance of void from conductor distorts highest electric field and lowest current density. Therefore, it is important to study both electric field and current density to ensure good capabilities and safety of HVDC cable.
\end{abstract}

Copyright () 2017 Institute of Advanced Engineering and Science. All rights reserved.

\section{Corresponding Author:}

N. A. M. Jamail,

Faculty of Electrical and Electronic Engineering,

Universiti Tun Hussein Onn Malaysia (UTHM),

86400 Batu Pahat, Johor, Malaysia.

Email: norakmal@uthm.edu.my

\section{INTRODUCTION}

In this study, it focuses more about simulation of HVDC cable insulation effects on the electric field. HVDC cable configuration of cross-linked polyethylene (XLPE). HVDC cable uses copper as a conductor. This HVDC cable was supplied by $11 \mathrm{kV}$.

The simulation of HVDC cable insulation effects on electric field and the current density was built up by using Quickfield software which is a powerful interactive environment for modeling and solving all kinds of scientific and engineering problems. Quickfield software also known as simulation software which designed to provide most accurate results when compare with real results.

This study begins with the understanding concept the importance of electric field and current density in high voltage engineering. The electric field is a quantitative description of the attraction or repulsion of one electric charge by another at any point[1]. HVDC configuration effects on electric field and current density can be determined by their configuration which provided the diameter of void and distance of void from conductor have been simulated by using Quickfield software [2-4].

From the HVDC cable configuration data obtained, a model of HVDC cable can be designed and show the result of maximum electric field(E) and current density(J). Each HVDC cable configuration will present different effect on electric field and current density.

Electrical and electronic equipment in the design and operation, knowledge of electric fields is vital for various applications. So, it is needed in high-voltage sources, machine windings and cables as well as within electronic equipment for the design of insulation and analyze electrical stresses. It is also used in the 
research of gas discharges and the effects of electric fields in their vicinity and in the design of ultra-high voltage substation. Electrostatic filters and xerography in industrial applications also use electric field [5-8].

Depending on how its produce, there are different kinds of current densities which are consist of convection current density, conduction current density and displacement current density. Conduction current requires a conductor. A conductor is characterized by a large number of free electrons that provide conduction current due to an impressed electric field, the force on an electron with charge -e when an electric field $\mathrm{E}$ is applied [9-10].

\subsection{Breakdown Due to Internal Discharge at Void}

Voids or cavities within the medium or at the boundaries between the dielectric and the electrodes is present in the solid insulating materials and to a lesser extent liquid dielectrics. These voids are generally filled with a medium of lower dielectric strength, and the dielectric constant of the medium in the voids is lower than the insulation. Hence, the electric field strength in the voids is higher than that across the dielectric. Therefore, even under normal working voltages, the field in the voids caused it to exceed their breakdown value, and breakdown may occur [11]. Solid insulators used will be subjected to high electric stress and very low temperatures. Electrical breakdown and degradation of insulator will be occurred when voids included in the solid insulators because of partial discharge [12].

Figure 1(a) show dielectric between two conductors. If we divide the insulation into three parts, an electrical network of $\mathrm{C}_{1}, \mathrm{C}_{2}, \mathrm{C}_{3}$ can be formed as shown in Figure 2(b). In this $\mathrm{C}_{1}$ represents the capacitance of the void or cavity, $\mathrm{C}_{2}$ is the capacitance of the dielectric which is in series with the void, and $\mathrm{C}_{3}$ is the capacitance of the rest of the dielectric [11-14].

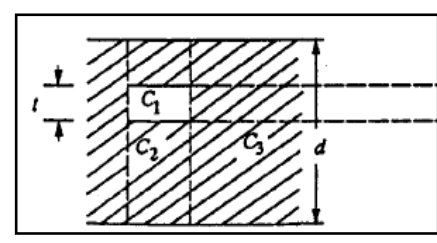

(a)

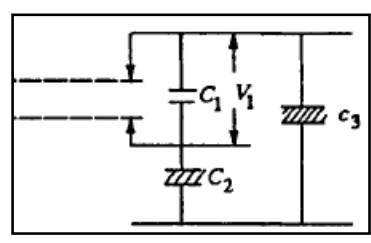

(b)

Figure 1. (a) Dielectric between two conductors (b) Formed of electrical network C1, C2, C

\subsection{Cross-linked Polyethylene (XLPE)}

XLPE is an abbreviated designation of "cross-linked polyethylene". XLPE is widely supplied for HVAC while HVDC cables, primarily use oil-immersed paper insulation [15]. Polyethylene has a linear molecular structure as shown in Figure 3(a) [16]. As shown in Figure 3(b), Molecules of polyethylene will be defect easily at high temperature when it is not chemically bonded.

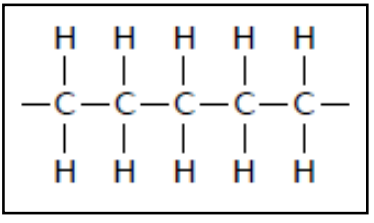

(a)

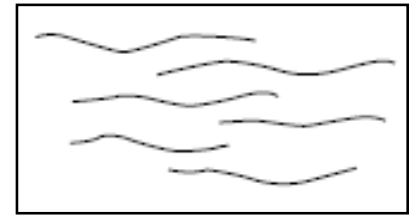

(b)

Figure 2. (a) Molecular structure of polyethylene (b) Chemically bonded of polyethylene molecules

A three-dimensional network in XLPE molecules bonded is shown in Figure 3(a) and Figure 3(b) deformation even at high temperature have strong resistance [16].

Cross-linked polyethylene is produced from polyethylene under high pressure with organic peroxides as additives. The cross-linking had been affected in the application of heat and pressure. In this application, the individual molecular chains had been linked with one another and causes the material change into an elastic material from a thermoplastic [16]. 
The dielectric loss is the main advantage of XLPE insulation for medium and high voltage cables. The dielectric loss factor of XLPE insulation is around one decimal power lower than paper insulated cables and around two decimal powers lower than that of PVC-insulated cable [17]. The mutual capacitance of XLPE cables is also lower and reducing the charging currents and earth leakage currents in networks without the rigid star point earthing when the dielectric constant is good [16]. Besides, XLPE is more conservative for submarine cables than for underground ground. So, XLPE insulator can reduce the repair cost [18].

Power transmission and distribution system especially in urban area had been widely used XLPE cables since about 40 years ago. Nowadays, some XLPE cables have been operated for about 30 years and it had been designed lifetime in their production. Hence, it is important to understand voltage withstand stress of aged XLPE cable to ensure reliable operation and minimized replacement planning for the aged XLPE cable [19-20]

The development of XLPE insulator for HVDC power transmission had increased the performance of the insulators. All of the characteristics and advantages of XLPE had been summarized in Table 2.

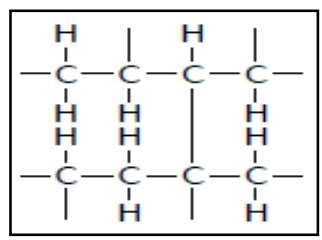

(a)

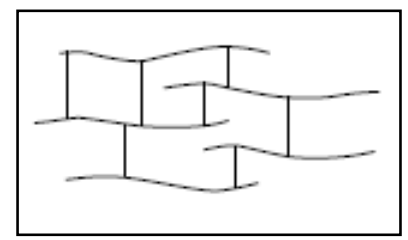

(b)

Figure 3. (a) Linear molecular structure of polyethylene (b) Chemically bonded of polyethylene molecules

Table 2. Characteristics and advantages of XLPE[4]

\begin{tabular}{|c|c|}
\hline Characteristics and Advantages of XLPE & Details \\
\hline Excellent Electrical and Physical Properties & $\begin{array}{l}\text { XLPE cable constitutes the best cable for transmission and distribution lines } \\
\text { because of its excellent electrical and physical properties. }\end{array}$ \\
\hline Capability of Carrying Large Currents & $\begin{array}{l}\text { The excellent resistance to thermal deformation and the excellent aging property } \\
\text { of XLPE cable permit it to carry large currents under normal }\left(90^{\circ} \mathrm{C}\right) \text {, emergency } \\
\left(130^{\circ} \mathrm{C}\right) \text { or short circuit }\left(250^{\circ} \mathrm{C}\right) \text { conditions. }\end{array}$ \\
\hline Ease of Installation & $\begin{array}{l}\text { XLPE cable withstands smaller radius bending and is lighter in weight, allowing } \\
\text { for easy and reliable installation. Furthermore, the splicing and terminating } \\
\text { methods for XLPE cable are simpler in comparison with other kinds of cables. }\end{array}$ \\
\hline $\begin{array}{l}\text { Free from Height Limitation } \\
\text { Maintenance }\end{array}$ & $\begin{array}{l}\text { XLPE cables can be installed anywhere without special consideration of the } \\
\text { route }\end{array}$ \\
\hline & $\begin{array}{l}\text { profile (height limitations) since it does not contain oil and thus is free from } \\
\text { failures due to oil migration in oil-filled cables. }\end{array}$ \\
\hline No Metallic Sheath Required & $\begin{array}{l}\text { XLPE cable does not generally demand a metallic sheath. Thus, it is free from } \\
\text { the failures peculiar to metallic sheathed cables, such as corrosion and fatigue. }\end{array}$ \\
\hline
\end{tabular}

\section{RESEARCH METHOD}

In this section, it is explained the research method to obtain the simulation results of electric field and current density of XLPE insulator by using Quickfield Software.

\subsection{Material Properties}

The conductor materials which is used for insulator are shown in Table 3 below. After that, the simulation is run based on these properties.

Table 3. Materials use for HVDC cable

\begin{tabular}{lll}
\hline Material & Relative Permittivity, $\varepsilon_{\mathrm{r}}$ & Conductivity, $\sigma(\mathrm{S} / \mathrm{m})$ \\
\hline Air & 1 & $3 \times 10^{-15}$ \\
Copper & 1 & $5.9 \times 10^{7}$ \\
XLPE & 2.25 & $4 \times 10^{-8}$ \\
\hline
\end{tabular}

Figure 4 shows HVDC cable profile and dimensions. The insulator is made from XLPE insulation. XLPE having a conductivity of $\sigma=4 \times 10^{-8}$. The conductor is copper supplied with $11 \mathrm{kV}$ voltage. And the 
shield is set as the ground which $0 \mathrm{~V}$. The insulation thickness is $3.4 \mathrm{~mm}$ or $7.389 \mathrm{~mm}$ from the center of the conductor. The radius of conductors is $3.989 \mathrm{~mm}$ and diameter of the void is varying from $0.4 \mathrm{~mm}$ to $1.6 \mathrm{~mm}$ while distance of void from the center of the conductor with constant diameter is vary from $4.7 \mathrm{~mm}$ to $5.9 \mathrm{~mm}$.

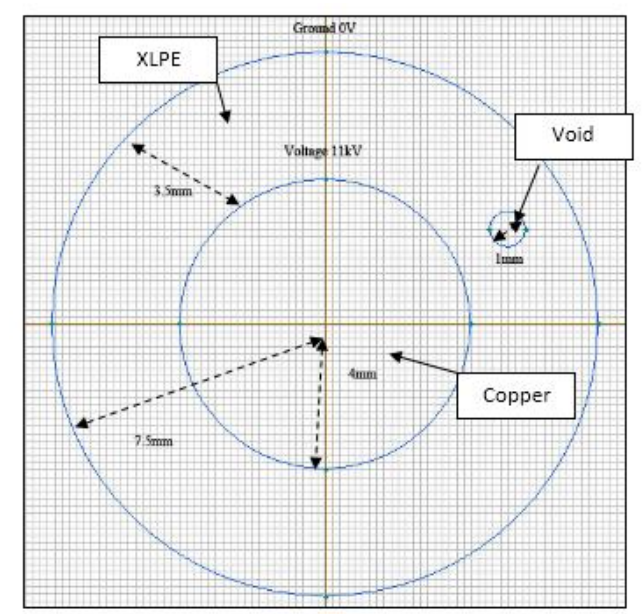

Figure 4. HVDC cable profile and dimensions

\subsection{Electric Potential and Ground}

The electric potential is $11 \mathrm{kV}$ and ground supplied is $0 \mathrm{~V}$. The parts where the electric potential was put in the software are shown in the Figure 5 and Figure 6. Figure 5 shows window for voltage label properties and boundary region for voltage. The voltage value is set by double click "Conductor" as labeled on the left of the screen and type $11000 \mathrm{~V}$ as $11 \mathrm{kV}$.

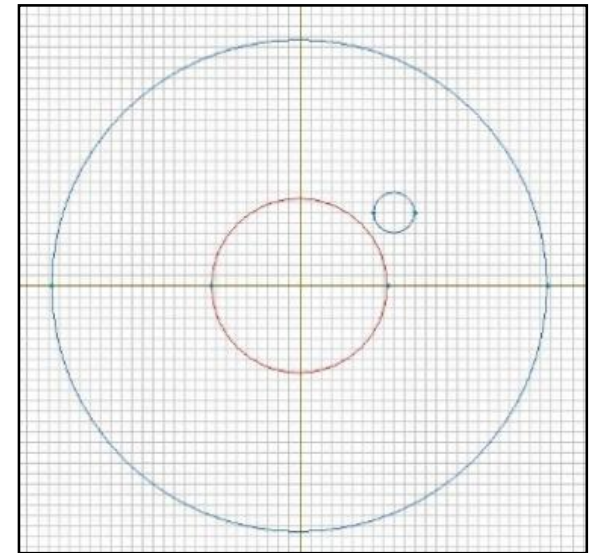

Figure 5. Boundary region for voltage

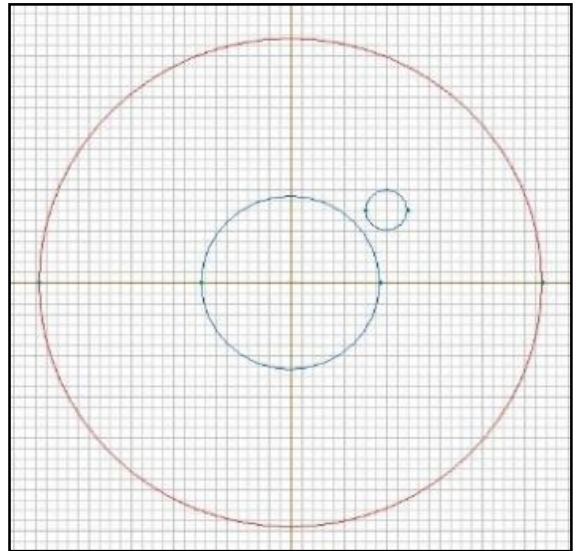

Figure 6. Boundary region for ground

Figure 6 shows window for ground label properties and boundary region for ground. At window for ground label properties, $0 \mathrm{~V}$ is typed at "Shield" labeled as ground.

\subsection{Mesh and Problem Solving}

The model will be converted into mesh after all of the material properties and boundaries are inserted to the HVDC cable by click "Build Mesh" icon on the Model toolbar as shown in the Figure 7. For the Student Version of Quickfield, the total number of nodes is limited to 250 only. 


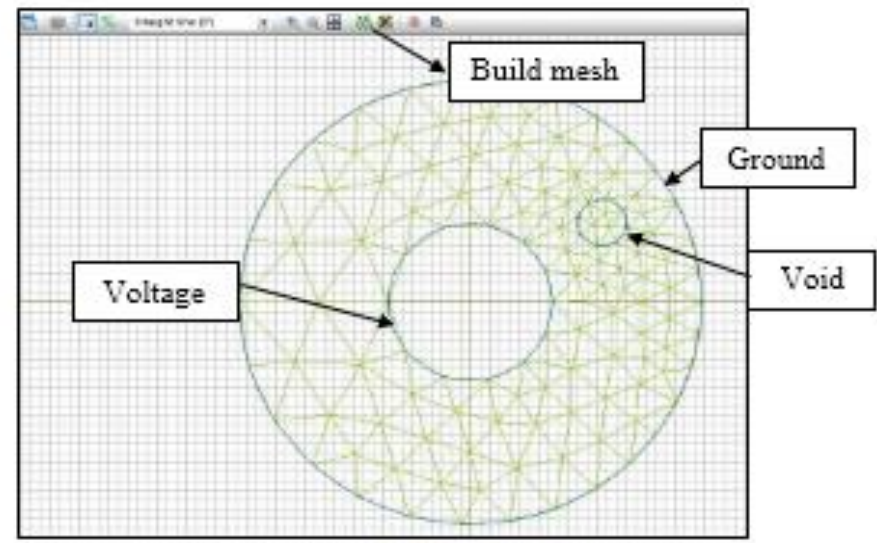

Figure 7. HVDC cable after convert into mesh

\section{RESULTS AND ANALYSIS}

\subsection{Simulation Results for Electric field and Current Density on XLPE Insulation}

Figure 8 shows that the electric field decreases from conductor to the shield of the insulator. This result can be viewed from the color map and the value referred to the legend as shown in. Results from HVDC cable which convert into the form color map shows that red colors indicate high voltage while blue colors indicate ground. It shows that color map pattern of current density also same with the color map pattern of the electric field.

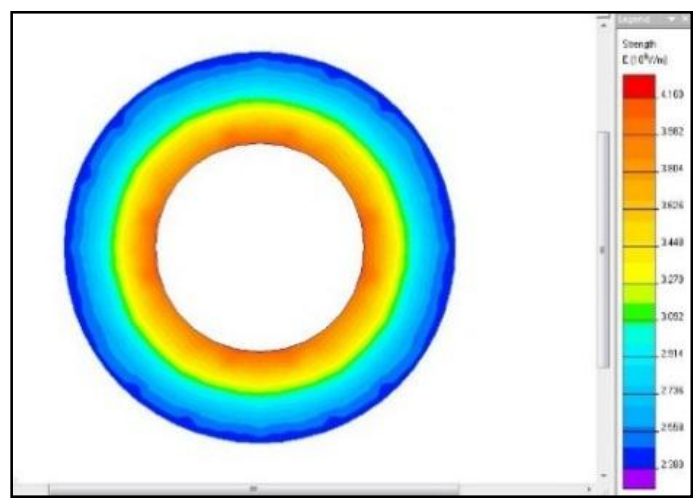

(a)

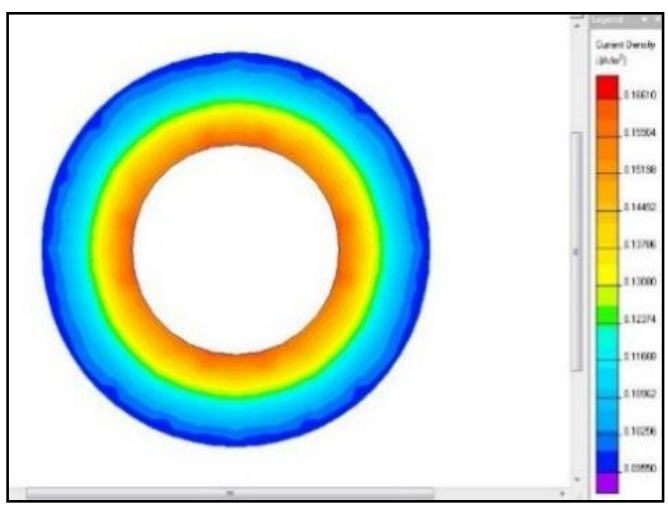

(b)

Figure 8. (a) Color map and legend of electric field strength in normal HVDC cable (b) Color map and legend for current density in normal HVDC cable

Figure 9 shows the electric field strength at normal HVDC cable without any defect. Based on Figure 9, the graph of the electric field and current density decreased gradually from $0 \mathrm{~mm}$ to $3.5 \mathrm{~mm}$ which is starting from conductor to the ground of cable. It shows that the electric field strength and a current density of insulation are highest when close to the conductor and become weaker when far from the conductor.

Table 4 shows the comparison between electric field and current densities of normal HVDC cable and defect HVDC cable. Maximum electric field $\mathrm{E}_{\max }$ at defect HVDC cable insulation is higher than $\mathrm{E}_{\max }$ at normal HVDC cable insulation. Maximum current densities $\left(\mathrm{J}_{\max }\right)$ at defect HVDC cable insulation is lower than $\mathrm{J}_{\max }$ at normal HVDC cable insulation. From these results, it can be concluded that normal HVDC cable insulation is better than defect HVDC cable insulation because void in cable insulation can cause distortion of the electric field and current density. Thus, it can cause damage and poor performance because cable structure is changed when the void is presence on the XLPE insulator. 


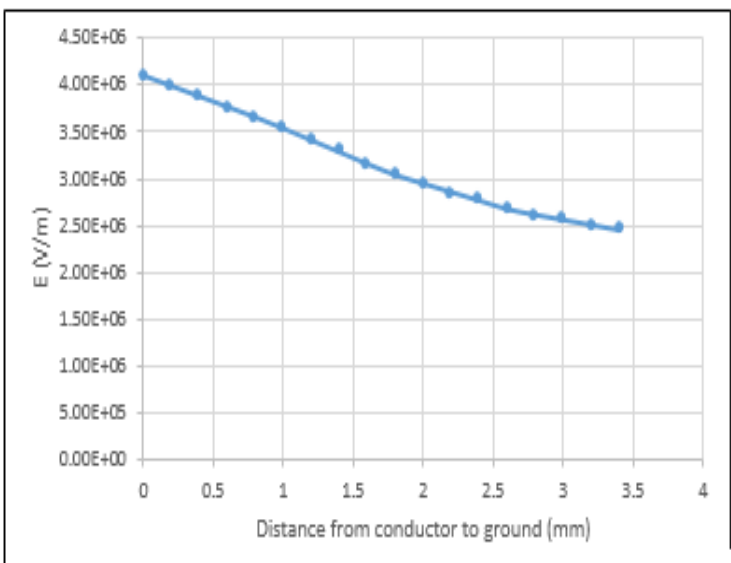

(a)

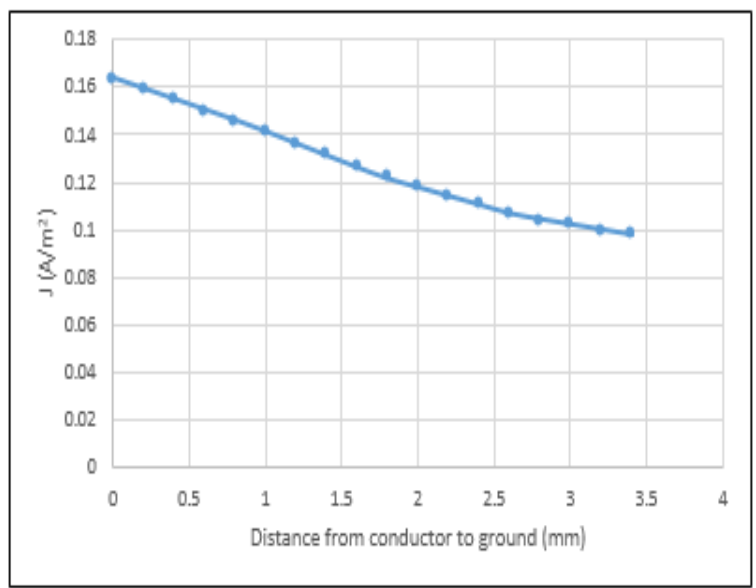

(b)

Figure 9. (a) Electric field strength at normal HVDC cable without any defect (b) Current densities at normal HVDC cable without any defect

Table 4. Comparison between electric field and current densities at normal and defect HVDC cable insulation

\begin{tabular}{ccccc}
\hline $\begin{array}{c}\text { Condition } \\
\text { Of HVDC }\end{array}$ & \multicolumn{2}{c}{ Electric Field, E $(\mathrm{V} / \mathrm{mm})$} & \multicolumn{2}{c}{ Current Densities, $\mathrm{J}\left(\mathrm{A} / \mathrm{m}^{2}\right)$} \\
cable & Minimum $\left(\mathrm{E}_{\min }\right)$ & Maximum $\left(\mathrm{E}_{\max }\right)$ & Minimum $\left(\mathrm{J}_{\min }\right)$ & Maximum $\left(\mathrm{J}_{\max }\right)$ \\
\hline Normal & & & & \\
Defect & 2446.58 & 4097.69 & $9.7863 \times 10^{-8}$ & $1.63907 \times 10^{-7}$ \\
\hline
\end{tabular}

\subsection{Simulation and Analysis of Electric Field and Current Density on Different Diameter of Void}

Figure 10 shows a graph of the electric field with various diameter of void on XLPE insulator. Based on the graph, the value of the electric field has been increased dramatically at each of void area and has been decreased drastically at the end of the void area. It shows that the larger diameter of the void will increase the value of the electric field and will distort the original value of the electric field. Thus, HVDC cable had become more danger and damaged when the diameter of void on the insulator is larger.

Figure 11 shows a graph of current density with various diameter of void on XLPE insulator. The graph shows that maximum current density is at the $0 \mathrm{~cm}$ which is near the conductor of the cable while current density will drop to almost zero at the void area and increased at outside area of the void.

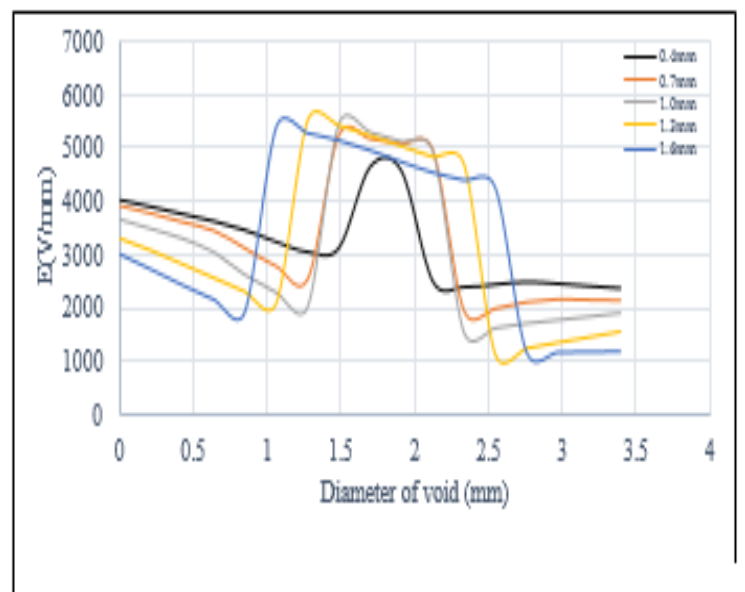

Figure 10. Electric field with various diameter of void

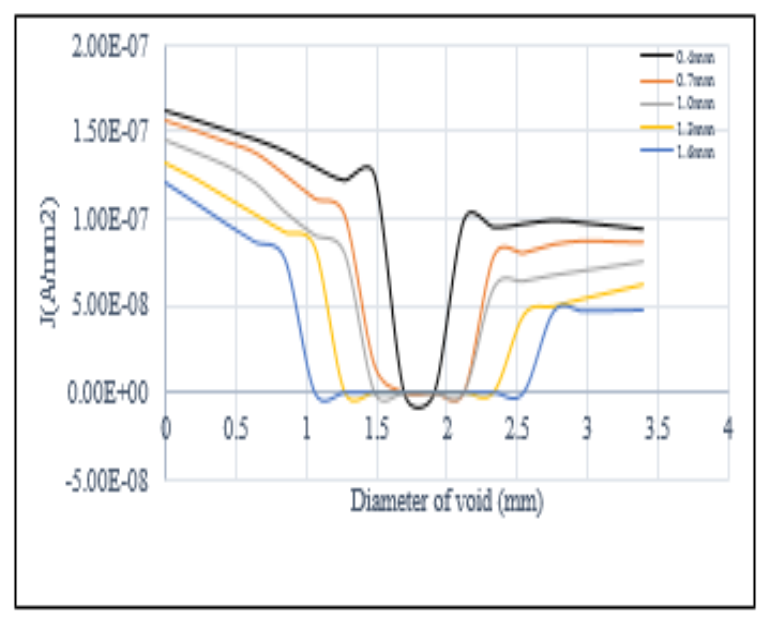

Figure 11. Current density with various diameter of void 
Table 5 shows the exact value of both electric field and current density on XLPE insulator. $\mathrm{E}_{\max }$ is obtained from the peak value of the electric field by referring to the Figure 10 and $\mathrm{J}_{\max }$ is obtained from the highest value of current density by referring to the Figure 11.

Table 5. Minimum and maximum of electric field and current densities with diameter of void

\begin{tabular}{ccccc}
\hline $\begin{array}{c}\text { Diameter of } \\
\text { void }(\mathrm{mm})\end{array}$ & \multicolumn{2}{c}{ Electric Field, E $(\mathrm{V} / \mathrm{mm})$} & \multicolumn{2}{c}{ Current Densities, J(A/mm $\left.{ }^{2}\right)$} \\
& Minimum $\left(\mathrm{E}_{\min }\right)$ & Maximum( $\left.\mathrm{E}_{\max }\right)$ & Minimum $\left(\mathrm{J}_{\min }\right)$ & Maximum $\left(\mathrm{J}_{\max }\right)$ \\
\hline 0.4 & 2345 & 4668.83 & $1.37 \times 10^{-14}$ & $1.61 \times 10^{-7}$ \\
0.7 & 1965 & 5227.31 & $1.49 \times 10^{-14}$ & $1.56 \times 10^{-7}$ \\
1.0 & 1512.92 & 5436.05 & $1.47 \times 10^{-14}$ & $1.45 \times 10^{-7}$ \\
1.3 & 1105.27 & 5501.08 & $1.40 \times 10^{-14}$ & $1.31 \times 10^{-7}$ \\
1.6 & 1165.01 & 5427.06 & $1.28 \times 10^{-14}$ & $1.21 \times 10^{-7}$ \\
\hline
\end{tabular}

Figure 12 shows a graph of $\mathrm{E}_{\max }$ with effect of different void diameter on XLPE insulator. The value of $E_{\max }$ has been increased when the diameter of the void is larger. It shows that larger diameter of the void will distort more original electric field and can cause higher risk and danger for HVDC cable.

Figure 13 shows a graph of $\mathbf{J}_{\max }$ with effect of different void diameter of void on XLPE insulator. Based on the graph, $J_{\max }$ is decreasing when the diameter of the void is larger. It shows that larger diameter of the void can decrease the capabilities of the insulator to conduct current density on the insulator.

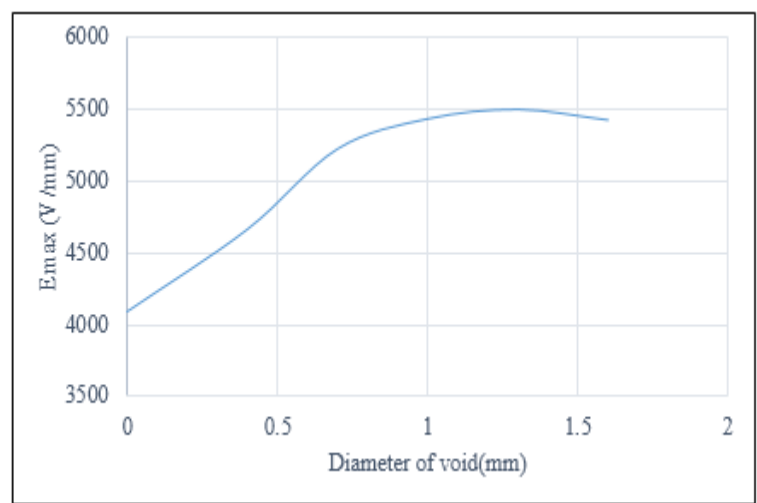

Figure 12. Emax value with effect of different void diameter

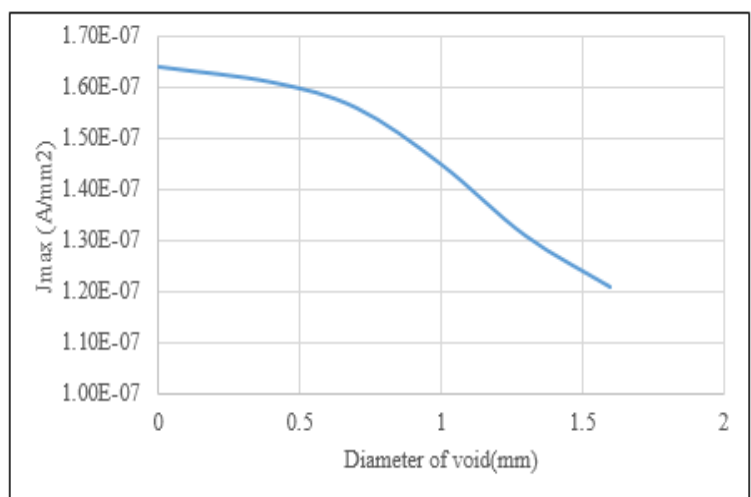

Figure 13. $\mathbf{J}_{\max }$ value with effect of different void diameter

\subsection{Simulation and Analysis of Electric Field and Current Density on Distance of Void from Conductor}

Figure 14 shows a graph of the electric field with various distance of void. Based on the graph, it shows that value of electric field at a void area slightly decreasing when the distance of void from the conductor is increased.

Figure 15 shows a graph of current density with various distance of void. Based on the results, the value of current density slightly decreasing when the distance of void from the conductor is increasing while the value of current densities at the void area were almost zero.

Table 6 shows the value of the electric field and current densities with the distance of void from the conductor is varied from $4.7 \mathrm{~mm}$ to $5.9 \mathrm{~mm}$. From the results, a distance of void at $47 \mathrm{~mm}$ from conductor has highest $\mathrm{E}_{\max }$ and lowest at $5.9 \mathrm{~mm}$ from the conductor. For maximum current densities $\left(\mathrm{J}_{\max }\right)$ when the distance of void is $4.7 \mathrm{~mm}$ from conductor simulate lowest $\mathbf{J}_{\max }$ and distance of $5.9 \mathrm{~mm}$ simulate highest value of current densities.

Figure 16 below shows a graph of $\mathrm{E}_{\max }$ value with effect of different distance of void from conductor. Based on the results, the value of $\mathrm{E}_{\max }$ is decreasing when the distance of void from the conductor is increasing which is further away from the conductor. It shows that electric field of HVDC cable will be more distorted when the presence of void is nearer to the conductor. 
Figure 17 shows a graph of $\mathbf{J}_{\max }$ against the distance of void from the conductor. The distance of void is also simulated starting from $0 \mathrm{~mm}$ which is the value of $\mathbf{J}_{\max }$ during normal condition. The insulator can carry current higher when the presence of void is further away from the conductor.

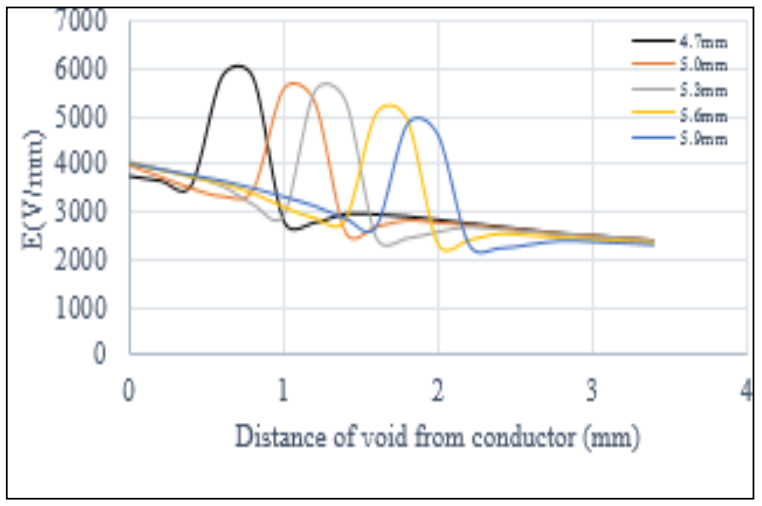

Figure 14. Electric field with various distance of void from conductor

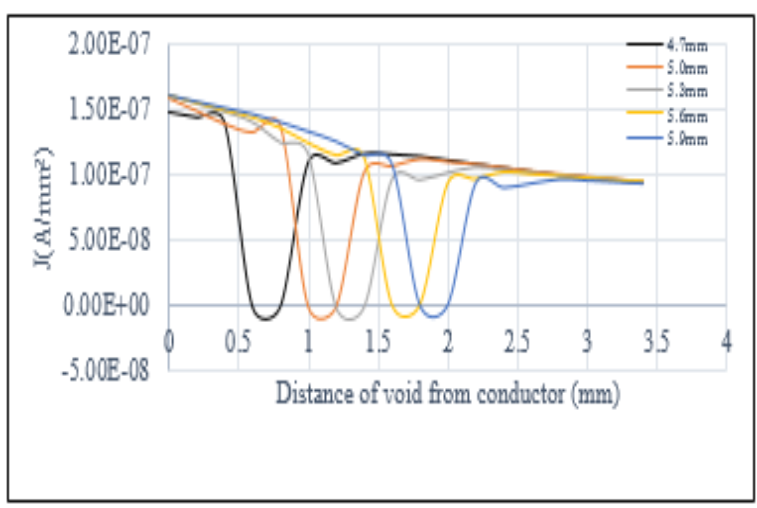

Figure 15. Current density with various distance of void from conductor

Table 6. Minimum and maximum of electric field and current densities with distance of void

\begin{tabular}{|c|c|c|c|c|}
\hline \multirow{2}{*}{$\begin{array}{l}\text { Distance of } \\
\text { void }(\mathrm{mm})\end{array}$} & \multicolumn{2}{|c|}{ Electric Field, E (V/mm) } & \multicolumn{2}{|c|}{ Current Densities, $\mathrm{J}\left(\mathrm{A} / \mathrm{mm}^{2}\right)$} \\
\hline & $\operatorname{Minimum}\left(E_{\min }\right)$ & $\operatorname{Maximum}\left(E_{\max }\right)$ & Minimum $\left(J_{\min }\right)$ & $\operatorname{Maximum}\left(\mathrm{J}_{\max }\right)$ \\
\hline 4.7 & 2388.76 & 5849.68 & $1.75 \times 10^{-14}$ & $1.5 \times 10^{-7}$ \\
\hline 5.0 & 2837.39 & 5599.22 & $1.60 \times 10^{-14}$ & $1.59 \times 10^{-7}$ \\
\hline 5.3 & 2391.9 & 5518.42 & $1.60 \times 10^{-14}$ & $1.62 \times 10^{-7}$ \\
\hline 5.6 & 2336.83 & 5063.35 & $1.48 \times 10^{-14}$ & $1.61 \times 10^{-7}$ \\
\hline 5.9 & 2264.4 & 4862.77 & $1.39 \times 10^{-14}$ & $1.62 \times 10^{-7}$ \\
\hline
\end{tabular}

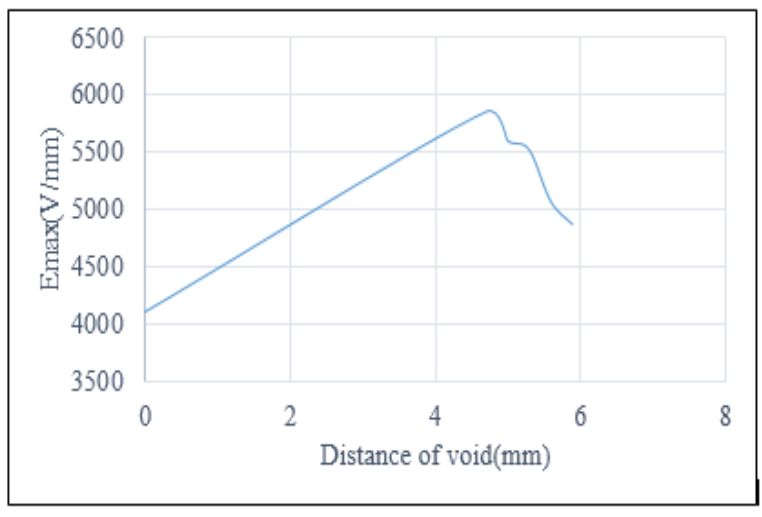

Figure 16. $\mathrm{E}_{\max }$ value with effect of different distance of void from conductor

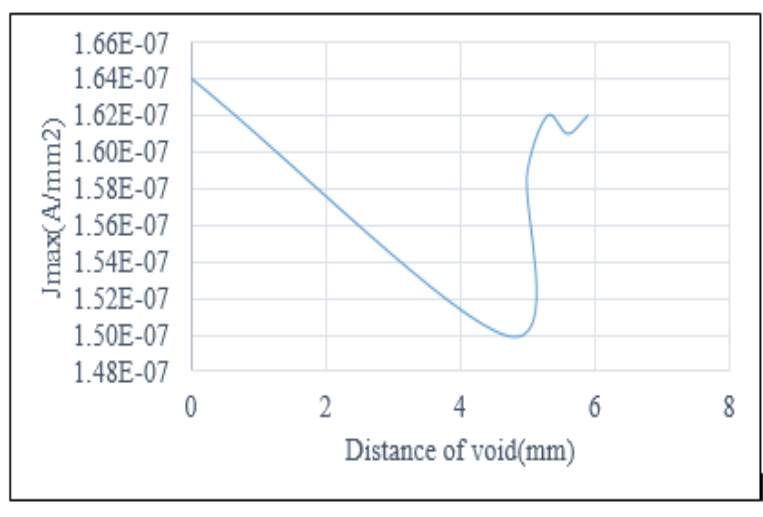

Figure 17. $\mathrm{J}_{\max }$ value with effect of different distance of void from conductor

\section{CONCLUSION}

By using this software, the XLPE cable have been analyzed which is maximum electric field $\left(\mathrm{E}_{\max }\right)$ is increased when diameter of void is increase and distance of void from conductor is decreased. While, maximum current density $\left(\mathrm{J}_{\max }\right)$ is decreased when diameter of void is decreased and distance of void from conductor is increased. Thus, the conclusion of this simulation can be identified.

For varying the diameter of the void, simulation results show that when the diameter of the void is increasing, it will produce a higher value of Emax and Jmax. This happens because conductivity of air is lower than conductivity of XLPE insulator. Therefore, the largest value of Emax will be produced at largest diameter of the void which is $1.6 \mathrm{~mm}$. For varying distance of void from the conductor, void at nearest 
distance from conductor produce highest $\mathrm{E}_{\max }$ and $\mathrm{J}_{\max }$. This is because the value of electric field produce near the conductor is higher and will become lower when further away from the conductor. Thus, nearest distance of void from conductor will produce the highest value of $\mathrm{E}_{\max }$ and $\mathrm{J}_{\max }$ which is at $4.7 \mathrm{~mm}$ from the conductor.

\section{ACKNOWLEDGEMENTS}

The authors gratefully acknowledge the Universiti Tun Hussein Onn Malaysia, Power and Renewable Energy Team (PaRENT), and High Voltage Laboratory UTHM for the equipment support.

\section{REFERENCES}

[1] R. Arora and W. Mosch, "High Voltage and Electrical Insulation Engineering," 2011.

[2] J. R. Claycomb, "Applied Electromagnetics Using Quickfield and Matlab," Infinity Science Press LLC.

[3] N. A. Othman, M. A. M. Piah, Z. Adzis, H. Ahmad, and N. A. Ahmad, "Simulation of voltage and electric-field distribution for contaminated glass insulator,” Proceeding - 2013 IEEE Student Conf. Res. Dev. SCOReD 2013, no. December, pp. 116-120, 2015.

[4] J. A. Morales, P. Gavela, N. Morales, and J. C. Montesdeoca, "Electromagnetic fields simulation on distribution feeders," 2015 IEEE NW Russia Young Researchers in Electrical and Electronic Engineering Conference (EIConRusNW). pp. 245-250, 2015.

[5] E. Bash, "High-Voltage Engineering Theory and Practice," PhD Propos., vol. 1, 2015

[6] F. Ulaby, E. Michielssen, and U. Ravaioli, "Fundamentals of Applied Electromagnetics," p. 528, 2010.

[7] W.Choo and G.Chen, "Electric field determination in DC polymeric power cable in the presence of space charge and temperature gradient under dc conditions," 2008.

[8] C. C. Reddy and T. S. Ramu, "On the computation of electric field and temperature distribution in HVDC cable insulation," Electr. Insul. IEEE Trans., vol. 13, no. 6, pp. 1236-1244, 2006.

[9] M. N. O. Sadiku, "Elements of Electromagnetics," no. 1887, pp. 3-6, 2010.

[10] D. Phenomena, S. H. Jayaram, and E. A. Cherney, "Calculation of current density," no. 3, pp. 375-378, 2003.

[11] M. S. Naidu, High Voltage engineering. McGraw-Hill.

[12] P. D. Degradation, S. Insulators, W. An, A. V. At, and L. Nitrogen, "Partial discharge degradation," vol. 10, no. 467, pp. 369-372, 1999.

[13] N. G. Trinh, "Partial discharge XIX: discharge in air part I: physical mechanisms," IEEE Electrical Insulation Magazine, vol. 11, no. 2. pp. 23-29, 1995.

[14] P. Liu, L. Cheng, H. Mei, and L. Wang, "Insulator with Internal Defect," pp. 1011-1014, 2015.

[15] N. Yoshifuji, T. Niwa, T. Takahasi, and H. Miyata, "Development of the new polymer insulating materials for HVDC cable," IEEE Trans. Power Deliv., 1992.

[16] Leader, "Xlpe Insulated Power Cable."

[17] Christian Folting, "Influence of insulation thickness on the electric Properties of unfilled $\mu \mathrm{m}$-sized thermosets."

[18] M. Muhr, E. Neges, R. Woschitz, and C. Sumereder, "Aging behaviour of cross-linked polyethylene (XLPE) as an insulating material for high (HV) - and extra-high voltage cables (EHV)," 17th Annu. Meet. IEEE Lasers ElectroOptics Soc. 2004. LEOS 2004, pp. 232-236, 2004.

[19] T. Takahashi, T. Kusaishi, T. Takahashi, H. Suzuki, and T. Okamoto, "SS-1 Development of Pre-breakdown Discharge Detection Test for Aged XLPE Cable to Find A Deteriorating Water Tree," vol. 2011, p. 2011.

[20] F. Duchateau, E. De France, and L. Renardikres, "of High Voltage Cables in France," pp. 27-30.

\section{BIOGRAPHIES OF AUTHORS}

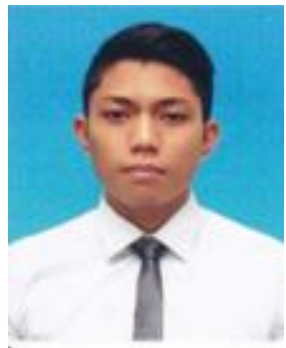

Muhammad Hariz Mohd Sharif is graduated in Bachelor of Electrical Engineering from Universiti Tun Hussein Onn Malaysia (2017). His researches are in fields of electrical engineering (power), high voltage, HVDC cable and Quickfield software. He is also involved in rowing sport. 

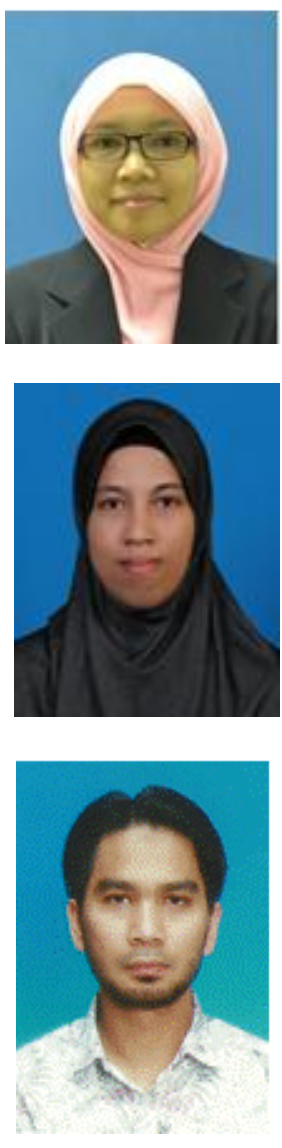

Nor Akmal Mohd Jamail is a lecturer of Universiti Tun Hussein Onn Malaysia since 2008. She received the Bachelor degree in Electrical Engineering from Universiti Teknologi Malaysia in 2005, M.Eng in Power System from Universiti Tun Hussein Onn Malaysia in 2007 and Ph.D. in High Voltage Engineering from Universiti Teknologi Malaysia, in 2015. Her research interests include condition monitoring of polymer nanocomposite insulation for HV purpose, dielectrics and electrical insulation. She is registered with the Board of Engineers Malaysia (BEM).

Nordiana Azlin Othman was born in Johor, Malaysia on January 19, 1986. She received Diploma in Electrical Communication and B.Eng in Electrical Engineering from Universiti Teknologi Malaysia (UTM) respectively in 2007 and 2010. She completed her PhD in Space Charge Distirbution at Institute of High Voltage and High Current (IVAT) in Faculty of Electrical Engineering, UTM. Her research interest includes the detection and diagnostics of partial discharge and space charge in insulation for condition monitoring.

M. S. Kamarudin obtained his B.Eng. and M.Eng. Degrees in Electrical Engineering (Power) from Universiti Teknologi Malaysia (UTM) in 2003 and 2005, respectively, and a Ph.D. in High Voltage Engineering from Cardiff University, UK, in 2014. Currently, he is a senior lecturer in the Faculty of Electrical \& Electronic Engineering at Universiti Tun Hussein Onn Malaysia (UTHM). His research interests include gas discharges, high voltage surge arresters, and dielectrics and electrical insulation system. He is registered with the Board of Engineers Malaysia (BEM). He is also currently a member of IEEE and a graduate member of Institution of Engineers, Malaysia (IEM). 